\title{
Proximity Operations for the Robotic Boulder Capture Option for the Asteroid Redirect Mission
}

\author{
David M. Reeves ${ }^{1}$ \\ NASA Langley Research Center, Hampton, VA., 23681, USA \\ Bo J. Naasz ${ }^{2}$ \\ NASA Goddard Spaceflight Center, Greenbelt, MD, 20771, USA \\ Cinnamon A. Wright ${ }^{3}$ \\ NASA Goddard Spaceflight Center, Greenbelt, MD, 20771, USA \\ Alex J. Pini ${ }^{4}$ \\ a.i. solutions, Inc., Lanham, MD 20706, USA
}

In September of 2013, the Asteroid Robotic Redirect Mission (ARRM) Option B team was formed to expand on NASA's previous work on the robotic boulder capture option. While the original Option A concept focuses on capturing an entire smaller Near-Earth Asteroid (NEA) using an inflatable bag capture mechanism, this design seeks to land on a larger NEA and retrieve a boulder off of its surface. The Option B team has developed a detailed and feasible mission concept that preserves many aspects of Option A's vehicle design while employing a fundamentally different technique for returning a significant quantity of asteroidal material to the Earth-Moon system ${ }^{1,2}$. As part of this effort, a point of departure proximity operations concept was developed complete with a detailed timeline, as well as $\Delta \mathrm{V}$ and propellant allocations. Special attention was paid to the development of the approach strategy, terminal descent to the surface, controlled ascent with the captured boulder, and control during the Enhanced Gravity Tractor planetary defense demonstration. The concept of retrieving a boulder from the surface of an asteroid and demonstrating the Enhanced Gravity Tractor planetary defense technique is found to be feasible and within the proposed capabilities of the Asteroid Redirect Vehicle (ARV). While this point of departure concept initially focuses on a mission to Itokawa, the proximity operations design is also shown to be extensible to wide range of asteroids.

\begin{tabular}{ll}
$\Delta \mathrm{V}$ & $=$ Change in velocity \\
$\mathrm{ACS}$ & $=$ Attitude Control System \\
$\mathrm{ARV}$ & $=$ Asteroid Redirect Vehicle \\
$\mathrm{ARRM}$ & $=$ Asteroid Redirect Robotic Mission \\
$\mathrm{CG}$ & $=$ Center of Gravity \\
$\mathrm{CRS}$ & $=$ Delta-Differential One-Way Ranging \\
Delta-DOR & Degree of Freedom \\
DOF & $=$ Enhance Gravity Tractor \\
$\mathrm{EGT}$ & $=$ Glash Pose \\
FPose & $=$ Ged Out Of Dodge \\
GNFIR & $=$ Specific Impulse \\
GOOD & \\
HP & \\
$\mathrm{I}_{\mathrm{sp}}$ & \\
\hline${ }^{1}$ Aerospace Engineer, Systems Analysis and Concepts Directorate, Senior Member AIAA. \\
${ }^{2}$ Project System Engineer, Satellite Servicing Capabilities Office, Senior Member AIAA \\
${ }^{3}$ Aerospace Engineer, Satellite Servicing Capabilities Office, Senior Member AIAA \\
${ }^{4}$ Aerospace Engineer, Satellite Servicing Capabilities Office
\end{tabular}




$\begin{array}{ll}\text { IWP } & =\text { Intermediate Waypoint } \\ \text { MFOV } & =\text { Medium Field of View } \\ \text { NEA } & =\text { Near Earth Asteroid } \\ \text { NFOV } & =\text { Narrow Field of View } \\ \text { PD } & =\text { Proportional Derivative controller } \\ \text { PoD } & =\text { Point of Departure } \\ \text { RCS } & =\text { Reaction Control System } \\ \text { SAA } & =\text { Sun }- \text { Asteroid }- \text { ARV angle } \\ \text { SAB } & =\text { Sun }- \text { Asteroid }- \text { Boulder angle } \\ \text { SEP } & =\text { Solar Electric Propulsion } \\ \text { SPC } & =\text { Stereophotoclinometry } \\ \text { TRN } & =\text { Terrain Relative Navigation } \\ \text { WFOV } & =\text { Wide Field of View }\end{array}$

\section{Introduction}

A key component of NASA's asteroid initiative is the Asteroid Robotic Redirect Mission (ARRM). One concept for the mission architecture that NASA is currently studying involves capturing a boulder, typically two to four meters in size, from the surface of a 100+ meter diameter Near-Earth Asteroid (NEA) and returning it to trans-lunar space $^{1}$. Additionally, one or more planetary defense demonstrations would be performed while at the target NEA. This concept provides NASA the option to trade potential return mass for centimeter level characterization of an entire large NEA, certainty of target NEA composition type, ability to select the boulder captured, additional experience operating in a micro gravity environment including extended surface contact, and the ability to demonstrate future deflection strategies on a relevant-sized NEA. This concept, called the ARRM Robotic Boulder Capture Option, or Option B, has been investigated to determine the mission feasibility and identify potential operational and vehicle capability differences from the initially proposed design of capturing an entire small asteroid, now referred to as Option $\mathrm{A}^{2}$.

The proximity operations design for the Option B includes identification and retrieval of a boulder from the surface of a larger asteroid, which is a key differentiator from Option A. Option B includes a relatively extensive characterization phase, the selection of a target boulder, descent to the surface, and ascent with the captured mass, all of which are not required by Option A. While this approach necessitates additional operational phases and more time in the vicinity of the NEA to complete capture of the target boulder, it also provides additional mission robustness and flexibility, as well as exploration, planetary defense, and science benefits.

A Point of Departure (PoD) concept of operations has been developed for a mission to Itokawa with a proximity operations architecture consisting of four major phases: approach and characterization, dry run sequence, boulder collection, and Enhanced Gravity Tractor (EGT) demonstration, which will be described in detail below. These operations have been designed to leverage experience from prior missions such as Hayabusa, NEAR Shoemaker, and DAWN and utilize existing technologies and operations developed for OSIRIS-Rex in order to reduce risk. Analysis shows that this concept is not only applicable to Itokawa, but is also robust to various NEA targets.

\section{Overview}

Tasked with defining a feasible mission architecture for the ARRM robotic boulder capture options, the ARRM Option B team was primarily centered at NASA Langley and Goddard Space Flight Centers but included members with varying expertise from other NASA centers and academia. Defining the operations around the parent asteroid and the details associated with the boulder collection were key factors in demonstrating the feasibility of the Option B concept and led the creation of proximity operations sub team.

The selection of Itokawa as an initial target case study offered a unique opportunity to develop the operations required for Option B on an asteroid that has already been visited by JAXA's Hayabusa sample return mission, which could be viewed as a precursor for the development of this design, providing high-resolution imagery, surface composition information, and gravity knowledge ${ }^{3}$. Itokawa is an attractive target for this mission because of its relatively slow rotation rate and the presence of thousands of identified boulders on the surface that are in the $2-5 \mathrm{~m}$ diameter size range, which fits within the Option B mission performance capabilities for return ${ }^{4,5}$. Mazrouei, et al. also identified smooth areas that cover $\sim 20 \%$ of Itokawa's surface, which provide a flat region with few hazards that would be suitable landing sites for a spacecraft the size of the Asteroid Redirect Vehicle (ARV). Focusing on these areas reduces the boulder candidate set, however there are still scattered boulders in these regions, leaving hundreds 
of potential targets that fit within the return capability. In the development of the PoD proximity operations design, a notional target was selected near the Hayabusa landing site in one of these smooth regions known as the Muses Sea.

With a baseline target on Itokawa selected, the proximity operations were developed and found to fit within a 400 day timeline including $\sim 50$ days of asteroid and boulder characterization, $\sim 70$ days for dry-runs and boulder collection, $\sim 260$ days for demonstration and verification of EGT technique, and $\sim 20$ days of margin. While this timeline is noticeably shorter than the proximity operations for other robotic asteroid missions (e.g. OSIRIS-REx and Hayabusa), ARRM Option B relies heavily on the experience gained from previously flown missions and the extensive research and planning that has already been conducted as part of future missions. Purposefully drawing from this knowledge minimizes mission-unique operations and therefore reduces risk. However, ARRM's fundamental objectives differ from these science-driven missions. While extensive amounts of scientific data and imagery will be obtained during the mission, ARRM Option B has a primary objective or collecting and returning a boulder from a NEA with a secondary objective of demonstrating a planetary defense technique. The timeline is constrained by the desire to have the boulder accessible to crew at a set timeframe. The demonstration of the EGT takes a considerable amount of time, which works against the return deadline in that as the total proximity operations timeline increases, the time for inbound Solar-Electric Propulsion (SEP) transfer is shortened, reducing the boulder mass the ARV is capable of returning.

The timeline consists of conservative operations in anticipation of the wide range of unknowns concerning the physical properties of the asteroid surface and potential target boulders. The dry-run sequence prior to boulder collection consists of two approaches that maximize the use of passively safe trajectories to achieve progressively lower altitudes, following the same path the ARV will take for the actually collection attempt. These dry-runs provide higher resolution imagery of the target boulder and surrounding area as well as a verification of the Terrain Relative Navigation (TRN) system. In order to account for a boulder that is unexpectedly buried, an unidentified soft spot in the asteroid regolith which provides unsteady footing for the ARV, an off-nominal collection that results in a fractured or crumbled boulder, or even a malfunction in the collection system, the timeline and propellant budget account for a total of three dry-run sequences and a total of five boulder collection attempts across those three sites.

Further protection against the uncertainty of the asteroid surface is provided by the attenuating Contact and Restraint System (CRS). During terminal descent below $20 \mathrm{~m}$, the ARV will only use horizontal thrusters and thrusters that plume away from the surface to maintain attitude and position over the boulder. The CRS will attenuate the residual contact velocity, which is dependent on the parent asteroid and boulder location, but is generally very low and for the Muses Sea region of Itokawa is found to be less than $5 \mathrm{~cm} / \mathrm{s}^{6}$. After the completion of the surface operations and successful boulder capture described by Merrill and Belbin, the CRS will then reverse the landing stroke that will break the cohesion between the boulder and the surface and provide an initial push off, which eliminates the need for any thruster pluming of the surface and potential creation of debris. For some boulder collection sites on asteroids that rotate faster than Itokawa, the centripetal force exceeds the gravitational force, requiring continuous downward thrust to make and maintain contact with the surface. This simplifies the problem in some ways by allowing the contact velocity to be controlled with thrust and providing ascent with no propulsion or mechanical push. After safely clearing the surface, the ARV will use Reaction Control System (RCS) thrusters to ensure escape velocity is reached and to remove the residual spin of the combined ARV and boulder system. Continuing on a slow drift trajectory, a series of small maneuvers will be performed to refine the estimated mass properties of the boulder and all boulder collection data will be downlinked prior to the transition to the EGT demonstration.

Preceding the start of the EGT demonstration, the ARV will maintain a distance of approximately $20 \mathrm{~km}$ from the asteroid for three weeks while the asteroid's orbit is more precisely determined. DSN range measurements from Earth to the ARV combined with relative range measurements from the ARV to the asteroid will provide an update of the asteroid's orbital parameters that will be used as reference for verifying the deflection. The ARV will then enter a non-Keplerian tractoring orbit (described in detail below) to demonstrate the EGT technique, followed by another stand-off period to allow the deflection to propagate and achieve favorable orbital alignment with Earth to conduct the ground-based deflection verification. The duration of the EGT demonstration depends on the size, mass, and orbit of the parent asteroid as well as the mass of the boulder collected. For Itokawa, assuming a $6,000 \mathrm{~kg}$ boulder is collected, it was found that 60 days in the tractor orbit followed by an additional 6 months of orbit propagation is sufficient to provide a measurable deflection beyond 3-sigma certainty. It was also found that if no boulder was collected, the EGT demonstration could still provide a measurable deflection by remaining in the tractoring orbit for 180 days followed by three months of orbit propagation. While the total duration does slightly increase if no boulder is successfully captured, the return date is not affected due to the lower total spacecraft mass on the return trajectory. Returning without a boulder would allow the crew to collect any surface samples or material 
that is on the CRS and provide a docking target in the Earth-Moon system for test missions. The ARV, with its large solar arrays, could potentially be used as a power source for future missions or even a SEP bus if refueled.

All of the above operations are dependent on the highly capable sensor suite of the ARV. The fully redundant sensor suite consists of three high definition cameras with differing fields of view and a 3D LIDAR. The Narrow Field of View (NFOV) and Medium Field of View (MFOV) cameras are located on a side mounted two-axis gimbal

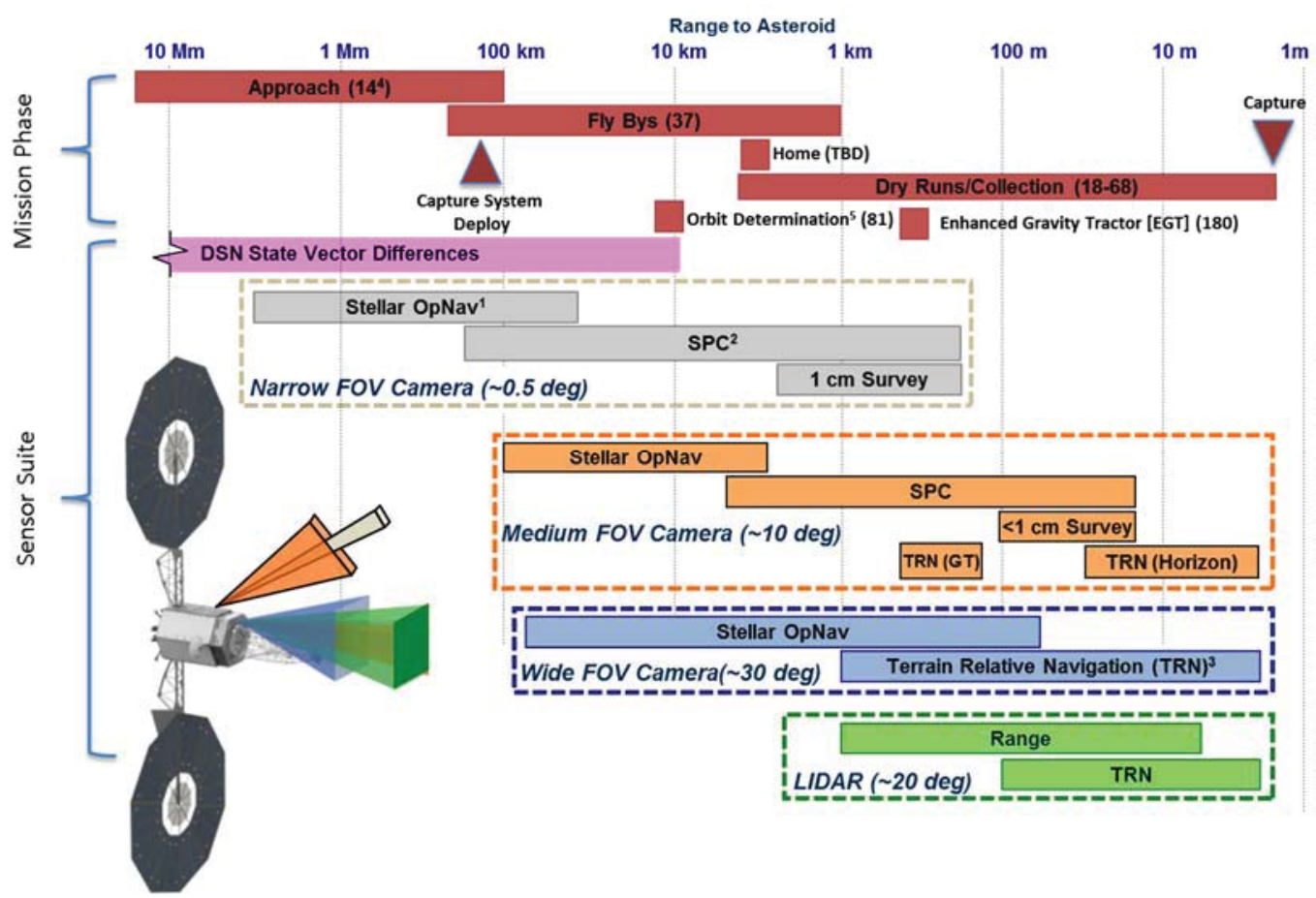

Figure 1. Option B Proximity Operations sensor suite

while the Wide Field of View (WFOV) and LIDAR are fixed to base of the capture system interface and are nadir pointing. Figure 1 depicts the sensor capabilities and architecture as it pertains to the proximity operations.

While Itokawa was selected as the first case study target, the operations were developed keeping three other potential targets in mind: Bennu, $1999 \mathrm{JU}_{3}$, and $2008 \mathrm{EV}_{5}$. All three of these additional targets are classified as carbonaceous as opposed to Itokowa's stony classification, which is considered more desirable due to the potential presence of hydrated material minerals and other volatiles. Bennu is the target of NASA's upcoming OSIRIS-REx mission and JAXA's Hayabusa II mission is targeting $1999 \mathrm{JU}_{3}$ which could both provide precursor data similar to Hayabusa for Itokawa, while $2008 \mathrm{EV}_{5}$ would be unvisited in the ARRM timeframe. These additional targets are currently being studied in support of the on-going risk reduction tasks for Option B with detailed operations targeted to be complete by the end of fiscal year 2014 .

\section{Approach and Characterization Phase}

The Approach and Characterization Phase is considered to be the first phase in the proximity operations sequence and begins on the interplanetary insertion trajectory. The first leg of this phase starts at a range of around $1,000 \mathrm{~km}$ where the incoming trajectory will follow a straight line with a 45-degree Sun-Asteroid-ARV (SAA) angle. At this range, a maneuver will be executed to target a waypoint that to $100 \mathrm{~km}$ from the center of the asteroid, with a time of flight of 14 days. During this time the lighting angle allows for good optical tracking by the NFOV camera in order to refine the asteroid's ephemeris and shape model as well update the asteroids attitude and spin states from the previous knowledge. In the case of Itokawa this prior knowledge is relatively precise, and would require only slight updates, but if the target is an unvisited asteroid with solely ground-based information to rely on, detailed shape and spin models may need to be developed in real time.

After the global asteroid characteristics are determined, detailed mapping of the surface, potential boulders, and gravity field is conducted through a series of fly-bys. The fly-bys, depicted in Figure 2, each have a close approach distance of about $1 \mathrm{~km}$ and close approach velocities decreasing from $1 \mathrm{~m} / \mathrm{s}$ to $0.1 \mathrm{~m} / \mathrm{s}$. Targeting different areas of 
the asteroid and different lighting conditions, the NFOV and MFOV cameras on the 2-axis gimbal will map the entire surface to identify landmarks to be used by the TRN system while targeting close approach imaging on potential boulder collection sites. During these close approaches the currently assumed cameras are estimated to provide $\sim 1 \mathrm{~cm}$ resolution of the potential target boulders and the surrounding terrain. There are, on average, seven days between each close approach to allow for downlink, ground processing, and uplink of targeting updates for the next fly-by.

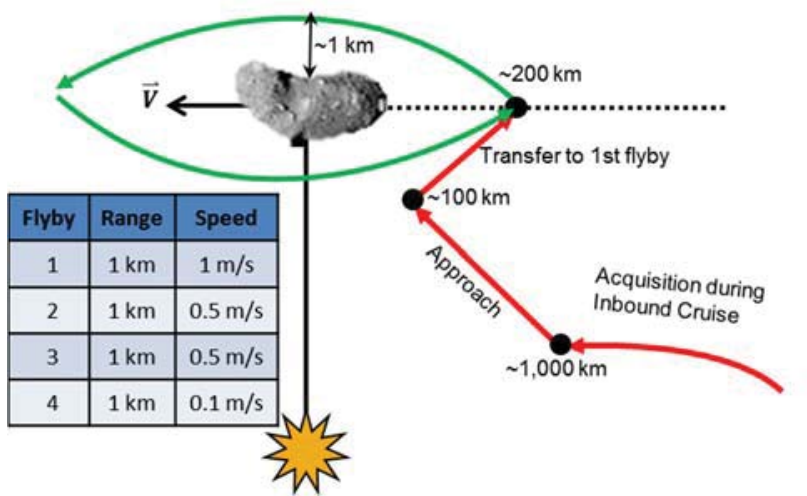

Figure 2. Approach and Characterization phase

Reducing the speed of the fly-bys allows more imaging time at higher resolution, but also helps to refine the knowledge of the gravity field. Based on imaging time and expected surface coverage, a series of four fly-bys was found to provide adequate imaging time and gravity knowledge for Itokawa and other targets which had precursor missions where preliminary characterization was conducted prior to the ARV's arrival. This allows the characterization fly-bys to be pre-designed to target selected regions and potential boulder locations. For previously unvisited NEAs, additional fly-bys would be needed to build gravity models and refine shape and spin models. They would also enable more close approaches over potential target boulders that were identified during acquisition and earlier fly-bys to provide higher resolution imagery. Once all fly-bys are complete, an additional week is reserved to gather any extra images requested by the ground team and to allow for final selection and prioritization of boulder collection targets.

\section{Dry-Run Sequence}

After three candidate boulders have been selected for capture and prioritized, a primary boulder location is chosen and the ARV enters a sequence consisting of two dry runs that bring the ARV incrementally closer to Itokawa. Once the dry runs have been successfully conducted, the ARV then proceeds to traverse through the same path towards the surface of the asteroid and attempts to collect the target boulder. The reason for executing the dry runs prior to the actual boulder collection attempt is to further map the surface features of the asteroid for optical navigational purposes, identify and rectify any operational issues, and initialize the on-board relative navigation filter. The dry runs are designed such that the ARV does not enter a potentially hazardous situation that could result in loss of satellite until the operations team has gained enough confidence to proceed.

The dry runs and final boulder collection attempt are made up of a series of waypoints whose locations are selected to accommodate certain design criteria, such as sensor ranges. The waypoints that comprise each of these sequences are presented in Table 1.

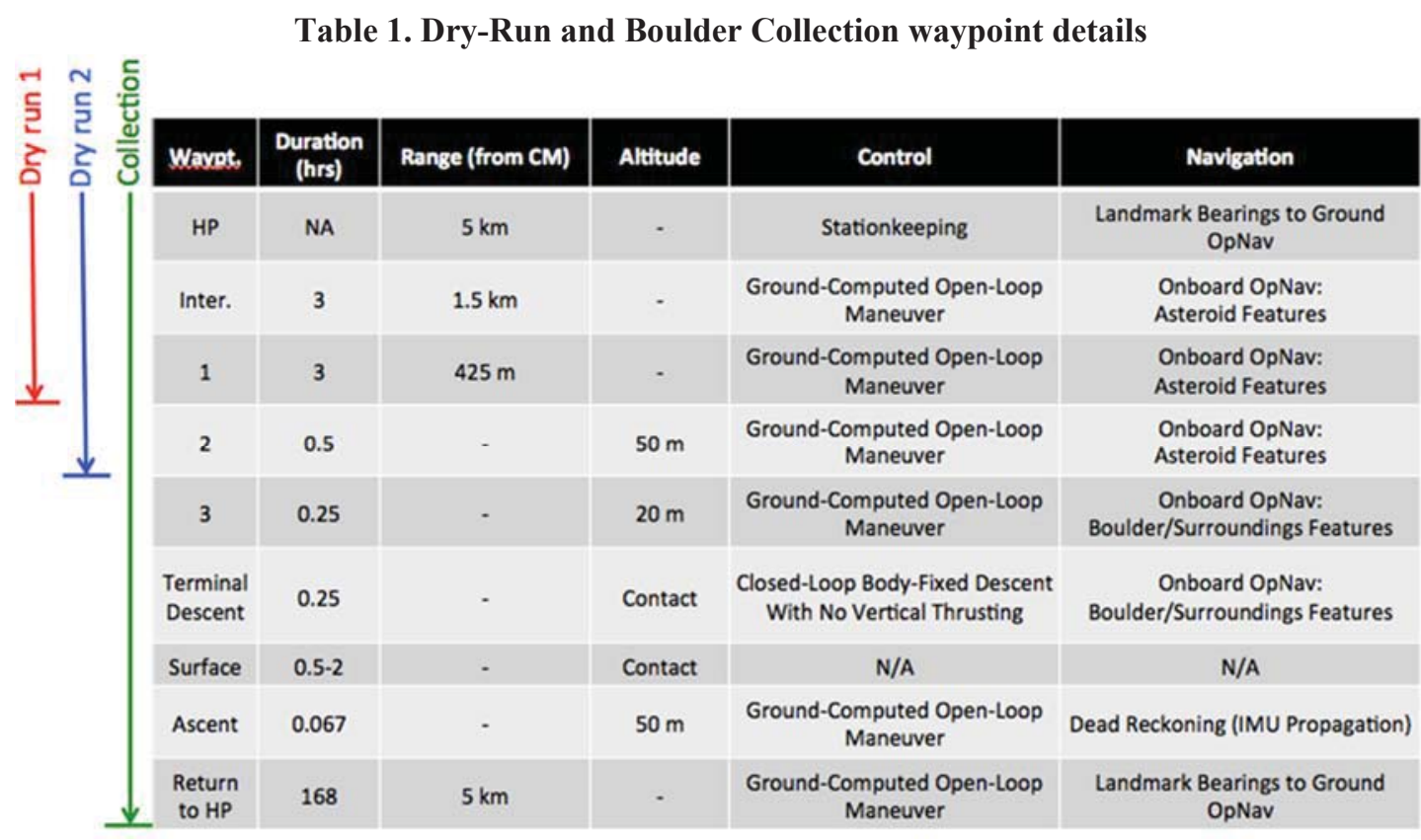


For ease of this preliminary analysis, each dry run begins at a reference point known as the Home Point (HP), which is located $5 \mathrm{~km}$ from Itokawa's center of mass. This waypoint was selected to be sufficiently far from the asteroid, yet in a location that would enable the observation of surface features with the ARV's NFOV and MFOV cameras. The HP should not be considered a safe hold point, but simply a reference for the beginning of each asteroid approach so that they each successive attempt follows the same path towards the asteroid. As a result, it is located in a region of space that is on the illuminated side of the terminator plane. Up to four open-loop maneuvers are computed on the ground prior to leaving this location, depending on which dry run sequence is being conducted.

From the Home Point, a ground-computed open-loop maneuver is performed to target the Intermediate Waypoint (IWP), which is located $1.5 \mathrm{~km}$ away from Itokawa's center of mass. This waypoint provides an indirect approach to the close proximity of the asteroid on a passively safe trajectory. If the ARV were to miss the maneuver that is planned to occur once the Intermediate Waypoint is reached, the spacecraft will drift safely away from Itokawa on a hyperbolic trajectory. Furthermore, this location offers a passively safe trajectory on the outbound leg to the next waypoint (Waypoint 1), so that a missed maneuver there will not result in a collision with the asteroid either. During the transit from the HP to the IWP, the ARV will continue to gather surface imagery of Itokawa via the NFOV and MFOV cameras.

Once the Intermediate Waypoint is reached, another ground-computed open-loop maneuver is immediately executed to send the ARV to Waypoint 1, which is located at a radius of $425 \mathrm{~m}$ from the asteroid center of mass. This distance was selected to ensure that the spacecraft was close to the surface of Itokawa without the threat of a potential collision due to an inaccurate estimate of the asteroid's orientation. Therefore, the waypoint is at a location that is about $100 \mathrm{~m}$ greater than the largest dimension of Itokawa. During the transfer from the Intermediate Waypoint to Waypoint 1, the ARV will enter the operational range of the LIDAR and WFOV camera, enabling those sensors to provide measurements that feed into the on-board relative navigation filter. Although the filter will be online at all times, the navigation solution will not be actively used until closed-loop descent is performed later in the sequence.

At Waypoint 1, a third ground-computed open-loop maneuver that is targeting Waypoint 2 is performed, sending the ARV to an altitude of $50 \mathrm{~m}$. This leg of the trajectory represents the first departure from being passively safe for a given sequence, as the spacecraft is now in the immediate vicinity of the asteroid. In addition to this waypoint being located $50 \mathrm{~m}$ above the surface of the asteroid, it is also directly above the boulder in that it lies on a line that is normal to the terrain local to the boulder site. An altitude of $50 \mathrm{~m}$ was selected to ensure that the spacecraft is sufficiently higher than the half-wingspan of the Megaflex arrays $(37 \mathrm{~m})$ that comprise the baseline hardware for this mission. This waypoint marks the transition between the open-loop ground-commanded maneuvers and the autonomous closed-loop continuous control that relies on the on-board relative navigation filter. With the last of four pre-computed open-loop maneuvers, the ARV matches the rotational rate of the asteroid and switches modes to assign the relative navigation filter as the prime source of information for the controller. Since the waypoint is located directly above the boulder when viewed from the surface and the ARV will be nadir-pointing at this time, the boulder will ideally be located directly in the bore sight of the LIDAR and WFOV camera when the closed-loop control commences.

With the spacecraft in closed-loop control mode, an appropriate continuous thrust profile will be calculated onboard to guide the ARV to Waypoint 3. This waypoint is also located along the local vertical line relative to the boulder and is $20 \mathrm{~m}$ above the surface of the asteroid. The final open-loop maneuver executed prior to this phase will help the spacecraft maintain the boulder in the field of view of the nadir-pointing sensors (LIDAR and WFOV camera) that are vital to the relative navigation during the moments before touchdown. The controlled descent from $50 \mathrm{~m}$ to $20 \mathrm{~m}$ will follow a "tapered" velocity profile that gradually reduces the vertical velocity relative to the surface down to nearly zero by the time Waypoint 3 has been reached. With the ARV still directly over the boulder at $20 \mathrm{~m}$, the terminal descent portion begins. During this phase the force of Itokawa's gravity will be allowed pull the spacecraft to the surface over the final distance. During terminal descent, use of the thrusters to slow the descent rate in the local vertical direction is disabled in an attempt to avoid pluming the target boulder and its surroundings, which could kick debris back into the spacecraft.

Although all of the waypoints listed above are contained within the final boulder collection attempt, the first two dry runs are versions of the final attempt that are truncated at different waypoints. The first dry run starts at the Home Point and progresses through Waypoint 1, at which point a "Get Out Of Dodge" (GOOD) maneuver is performed to send the spacecraft safely away from the asteroid. The purpose of GOOD maneuvers is to ensure that the ARV leaves the vicinity of Itokawa and to allow time for data downlink, model updates, and other operational tasks. The GOOD maneuvers can also serve as pre-computed abort maneuvers to be used at any point in the dry runs or final collection attempt should certain conditions be met to indicate an abnormal approach. After two days of drifting away from the asteroid, the ARV will maneuver to target the Home Point and begin the second dry run. 
During these two days, the spacecraft will have traveled a few dozen $\mathrm{km}$. This range depends on a trade between the $\Delta \mathrm{V}$ required to return to the Home Point and the certainty of leaving the vicinity of the asteroid. On the return trajectory to the Home Point, approximately three days of flight time allow for more operational duties to be performed, including the ground computation of the next four open-loop maneuvers on the upcoming second dry run.

Once the Home Point is reached, the second dry run commences and the ARV traverses through the same sequence of waypoints as the first dry run. However, once Waypoint 1 is reached, the spacecraft continues to Waypoint 2 rather than performing a GOOD maneuver. At Waypoint 2, the ARV will nominally be located directly above the target boulder and will have the boulder in the center of the field of view of its nadir-pointing sensors (LIDAR and WFOV camera). Here, the spacecraft performs its fourth open-loop maneuver and then initiates a controlled hover to maintain its position in the body-fixed reference frame to be directly above the boulder as the asteroid rotates. This hover will last for ten minutes and will serve as an opportunity to verify landmark identification and performance of the closed-loop on-board TRN system. After ten minutes of holding has elapsed, another GOOD maneuver is conducted to place the ARV on a similar escape trajectory from Itokawa. This GOOD maneuver and return leg have comparable shapes, purposes, and durations as those for the first dry run.

Once the Home Point is reached again and the operations team is confident that the environment is sufficiently characterized, the relative navigation filter is working properly, and the spacecraft systems are cleared to proceed, the final collection attempt will commence. For this sequence, all of the waypoints listed in Table 1 will be visited, with closed-loop descent beginning at Waypoint 2. At Waypoint 2 on the final collection attempt, the mission transitions into the Descent Phase, which involves the controlled descent from a $50 \mathrm{~m}$ altitude to the surface and will be outlined in detail below.

Since optical navigation is a critical part of the mission, all of these waypoints are located in regions that will provide a vantage point to look down on an illuminated portion of Itokawa's surface with favorable lighting conditions. As a result, every waypoint is located on the sun-lit half of the asteroid's terminator plane, as shown in Error! Reference source not found.. For waypoints in which the on-board TRN capability is actively used, which is Waypoint 2 and later, special considerations need to be made to ensure that the shadows cast by the target boulder and surrounding features are not too long, which may encumber the navigation algorithm. This corresponds to asteroid orientations where the target boulder is at or near the terminator plane, resulting in a Sun-Asteroid-Boulder (SAB) angle of roughly +/- 90 degrees. Furthermore, the ARV should avoid dwelling near the sun-line (an SAA of 0 degrees) during these later waypoints because doing so will cast the spacecraft's shadow over some or all of the target surface. As a result, these waypoints possess SAA values (and since the ARV hovers above the boulder, SAB values) between 30 and 45 degrees. These angles are maximized within the previously stated constraints to allow for the most illuminated operations time on the surface while the boulder site rotates towards the asteroid's heliocentric velocity vector and eventually back into darkness.

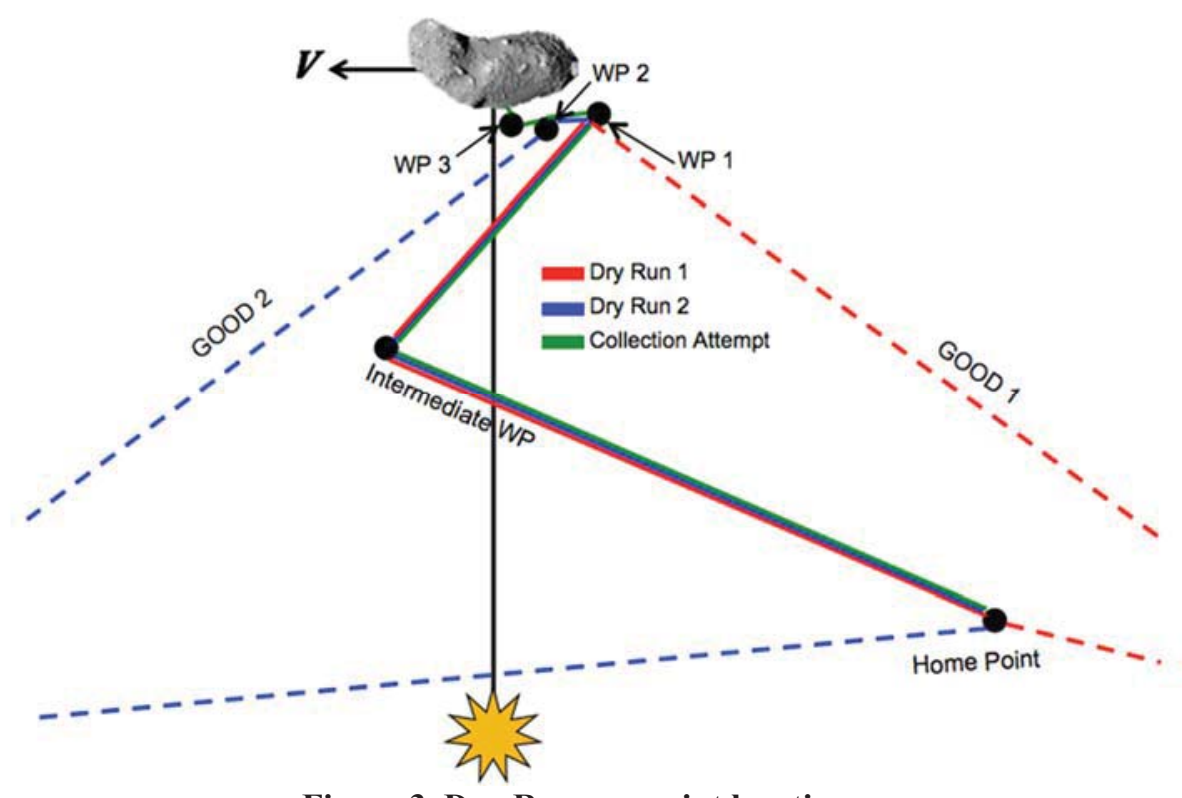

Figure 3. Dry-Run waypoint locations 
To obtain estimates of the $\Delta \mathrm{V}$ required to perform this sequence, the dry run phase has been simulated in FreeFlyer, a commercial simulation and mission design tool developed by ai Solutions, Inc. With the waypoint locations and times of flight between waypoints specified by the user, the simulation calculates the magnitude and three-dimensional direction in which an open-loop impulsive maneuver would need to be conducted at each waypoint to reach the next waypoint, assuming a ballistic coast between the two points. Although not all of the maneuvers in this phase are performed in this manner, this technique serves as a good first-order approximation for the closed-loop propellant requirements. This simulation also assesses the effect of the sensitivity of the dynamic environment around small celestial bodies on the ability to target waypoints. The modeling details associated with the simulation are outlined in Table 2.

Table 2. Dry-Run simulation parameters

\begin{tabular}{|c|c|c|}
\hline & Dry Run Simulation Parameters & \\
\hline Parameter & Value & Units/Notes \\
\hline Start Epoch & May 30, $202000: 00: 00$ & - \\
\hline NEA Gravitational Parameter ${ }^{7}$ & $2.36 \times 10^{-9}$ & $\mathrm{~km}^{3} / \mathrm{s}^{2}$ \\
\hline Central Body Gravity Modeling & Itokawa & Point Mass \\
\hline Third-Body Perturbations & Sun and Earth & $\mathrm{m}^{2}$ \\
\hline SRP Area & 250 & - \\
\hline$C_{R}$ & 1.4 & XYZ (m), asteroid-fixed coord. \\
\hline Boulder Location $^{7}$ & $126.27,76.77,-12.16$ & hours (simplified from 12.132)
\end{tabular}

A graphical representation of the baseline trajectory for the Itokawa dry run phase is illustrated in Figure 4 below. The RIC coordinate frame is used in the plots in Figure 4, with the Radial component defined by the Sun to asteroid position vector, the Cross-track component aligned with the asteroid's orbital angular momentum vector, and the In-track component completing the right-handed set in the general direction of the asteroid's heliocentric velocity vector.
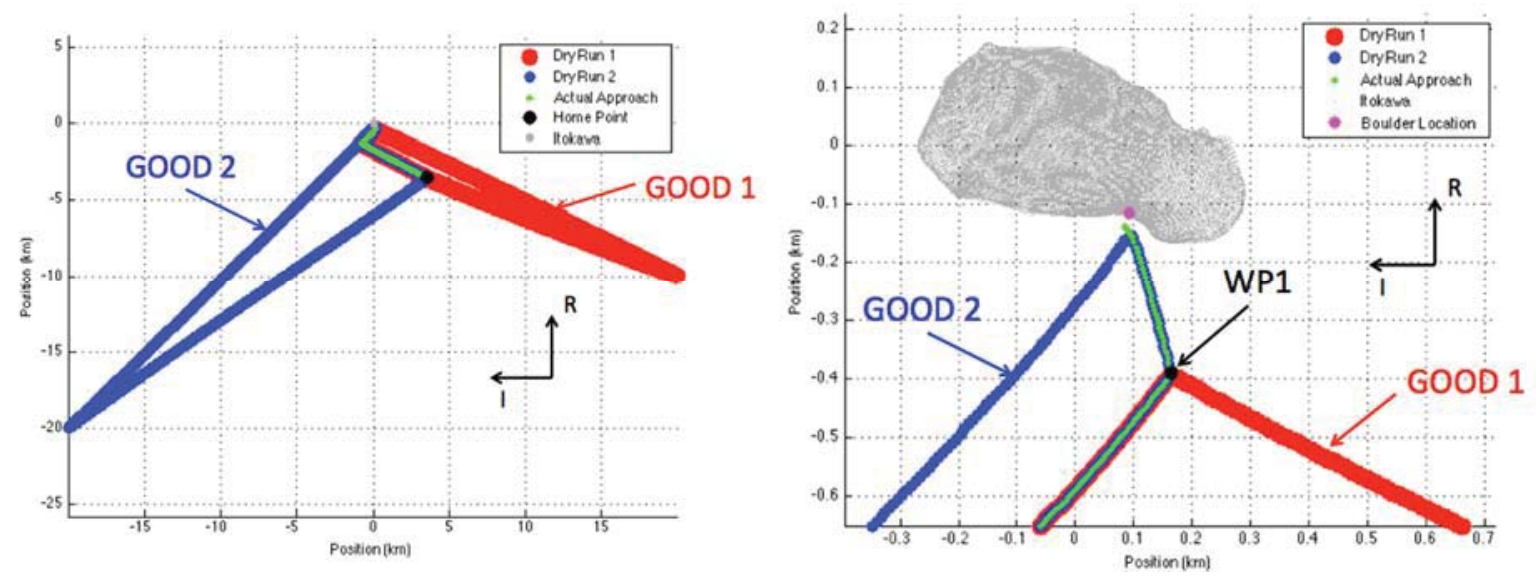

Figure 4. Dry-Rrun phase baseline trajectory

As mentioned previously, the locations of the later waypoints in the dry run phase are strongly dependent on the lighting conditions that the waypoint has to offer. As a result, the rotation rate of the target asteroid has a significant effect on the location of these waypoints, as the time of flight between the waypoints is currently fixed. For an asteroid such as Itokawa, which has a relatively long rotational period of around 12 hours, the ARV has ample time on the surface for operations. However, asteroids like Bennu and $2008 \mathrm{EV}_{5}$, with rotational periods of roughly 4 hours, provide a more challenging environment to land on and collect a boulder, as the illumination time, and thus 
the operational timeline, is condensed. Depending on the target asteroid's characteristics, this may necessitate a change in waypoint locations to provide more time for asteroid body-fixed descent and landing.

\section{Descent}

The boulder collection sequence follows the same path as the second dry run to Waypoint 2, where the terminal descent begins. At the start of this phase, which begins at an altitude of $50 \mathrm{~m}$, autonomous closed-loop control will be used to descend to the surface of the asteroid. At an altitude of $20 \mathrm{~m}$, the ARV nulls out its vertical velocity and finishes the terminal descent with no nominal thrusting in the direction of the surface to avoid pluming the target boulder and surroundings. Using horizontal RCS thrusters and fly-wheels, the ARV maintains a local vertical attitude while allowing gravity to slowly pull it towards the surface. Depending on the exact landing location on Itokawa the descent from $20 \mathrm{~m}$ will take approximately 15 minutes and result in a vertical contact velocity near 4 $\mathrm{cm} / \mathrm{s}$ which will be nulled by the attenuating contact arms of the capture system.

Optical cameras and LIDAR will be used to determine the state of the ARV relative to the boulder. Stereophotoclinometry (SPC) will be used to process images taken by the camera and generate an estimate of the spacecraft state using landmarks on the asteroids surface ${ }^{8}$. SPC is computationally intensive and will be performed on the ground while a separate TRN algorithm will operate onboard the ARV to provide real-time six-DOF navigation. GSFC's FlashPose (FPose) algorithm will be used to process real-time flash LIDAR frames to produce a six-DOF pose estimate ${ }^{9}$. Redundant TRN algorithms will operate simultaneously during the descent using the MFOV camera, the WFOV camera, and LIDAR.

A linear covariance analysis is currently being performed for the terminal descent to assess the performance of this system. The FPose measurement model used was

$$
y_{i}\left(t_{k}\right)=x_{i}\left(t_{k}\right)+b_{\text {constant }, i}+b_{\text {markov }, i}\left(t_{k}\right)+\eta_{i}\left(t_{k}\right)
$$

for each range in $\mathrm{x}, \mathrm{y}, \mathrm{z}$ and roll (about boresight), pitch and yaw. $y_{i}\left(t_{k}\right)$ is GNFIR's measurement at time $t_{k}, x_{i}\left(t_{k}\right)$ is the true displacement, $b_{\text {constant }, i}$ is a constant bias, $b_{\text {markov, } i}\left(t_{k}\right)$ is a Markov bias, and $\eta_{i}\left(t_{k}\right)$ is zero-mean Gaussian white noise with variance $\sigma_{\eta}^{2}$. The Markov bias is given by

$$
b_{\text {markov }, i}\left(t_{k}\right)=\alpha_{i} b_{\text {markov }, i}\left(t_{k-1}\right)+v_{i}\left(t_{k}\right)
$$

where $v_{i}\left(t_{k}\right)$ is zero-mean Gaussian white noise.

The optical navigation model estimates the pixel $s$, and line $l$, coordinates of the asteroid landmarks in the image. These are given by

$$
\left(\begin{array}{l}
S \\
l
\end{array}\right)=\left(\begin{array}{cc}
K_{x} & K_{x y} \\
K_{y x} & K_{y}
\end{array}\right)\left(\begin{array}{l}
x^{\prime} \\
y^{\prime}
\end{array}\right)+\left(\begin{array}{l}
S_{o} \\
l_{o}
\end{array}\right)
$$

where matrix $\mathbf{K}$ contains the reciprocal of the pixel dimensions, $s_{o}$ and $l_{o}$ are the zero pixel and line locations, and

$$
\left(\begin{array}{l}
x^{\prime} \\
y^{\prime}
\end{array}\right)=\left(\begin{array}{l}
x \\
y
\end{array}\right)+\left(\begin{array}{lll}
x r^{2} & x y & x^{2} \\
y r^{2} & y^{2} & x y
\end{array}\right)\left(\begin{array}{l}
\epsilon_{1} \\
\epsilon_{2} \\
\epsilon_{3}
\end{array}\right)
$$

where $\mathrm{x}$ and $\mathrm{y}$ are the coordinates in the image plane, as shown in Figure $\mathrm{xx}^{10}$. This model was included in NASA Goddard's Orbit Determination Toolbox (ODTBX) with assistance from Kenneth Getzandanner and is being incorporated into Draper and NASA JSC's Linear Covariance Analysis (LCA) tool for future analysis.

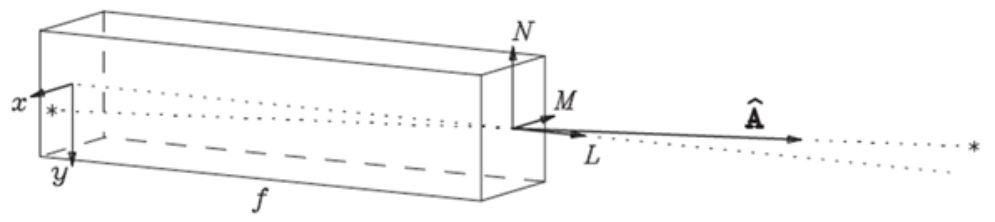

Figure 5. Pinhole camera showing gnomonic projection ${ }^{10}$ 
The ARV, sensor locations, and capture arms are shown below in Figure 6. On descent the capture arms will be pointed downward over the boulder and the LIDAR and WFOV sensors will be pointed downward, directly over the boulder. The MFOV sensor will be gimbaled and looking at landmarks on the asteroid. Ongoing analysis is being performed to quantify the performance of the GNC system using the LCA tool mentioned above.

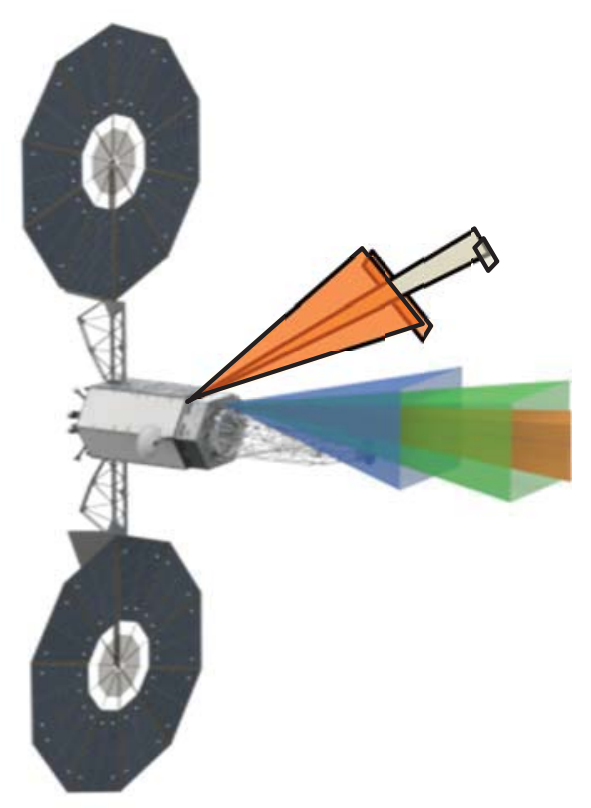

Figure 6. Sensor locations on the ARV

\section{Surface Operations and Ascent}

Given the landing uncertainty defined by the above analysis, the CRS will be prepositioned in a pose that will allow clearance around the boulder with a 3-sigma landing error. As described by Merill and Belbin, contact sensors located on the CRS pads will trigger a pre-planned attenuation profile to remove the residual velocity and bring the ARV to a stationary position over the target boulder. On Itokawa, the gravitational acceleration is greater than the centripetal acceleration, meaning the ARV can maintain this position in the absence of external forces. However, this gravitational force is small and the low resulting ARV weight is not sufficient to prevent tipping during the boulder capture. In order to provide more stability, four body-axis RCS thrusters will be pulsed to provide a low level of thrust, pushing the ARV into the surface for the duration of the surface operations. The ACS will remain active and if any unexpected motion is detected, the thruster pulse rate will be adjusted to increase the net downward thrust and stabilize the ARV. Thrusting into the surface allows the nominal concept of operations to be extensible to faster rotating asteroids with only the thrust level needing to be adjusted as the centripetal acceleration increases. In an effort to not excite any structural frequencies, the pulse pattern will be randomized.

With the ARV stabilized on the surface, the boulder is captured through a series of autonomous motions that are planned taking into account the boulder shape, the expected ARV landing pose, and the expected landing error ${ }^{6}$. The CRS will then perform a mechanical push-off to break cohesion and provide initial ascent. The push-off is designed to provide the combined ARV and boulder system with at least enough initial velocity to attain a $50 \mathrm{~m}$ altitude with the RCS system providing the rest of the impulse to exceed escape velocity. However in many cases, depending on the cohesion between the boulder and the surface, the CRS can provide enough impulse to achieve escape velocity from these low gravity bodies. 


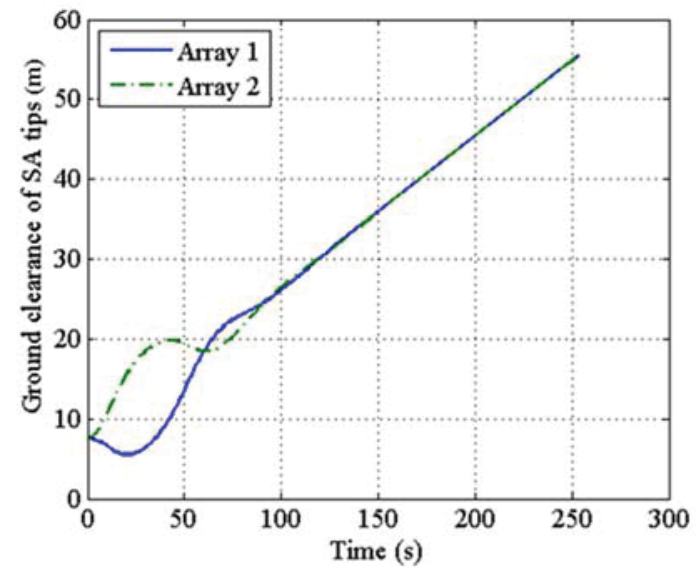

Figure 7. Solar array ground clearance for worst case ascent uncertainty. Case shown includes a $40 \mathrm{t}$ boulder with a horizontal cg offset that is $50 \%$ of the boulder radius and combined cohesion and uneven CRS push-off force that is twice the nominal CRS push-off force. Both forces are aligned with the solar arrav axis for worst case.

equal to twice that of the nominal CRS push-off along the solar array axis. These combined variances to the nominal ascent produce a tipping moment well beyond what would be expected, yet are still within the nominal control authority of the ARV, which demonstrates feasibility of the operations and design and robustness to the outstanding uncertainties.

After ascending from the surface, a small RCS burn is used to remove the residual spin from the motion of the asteroid surface and the spacecraft continues to coast away from the asteroid while holding an inertial attitude with both sun and Earth line of sight ${ }^{11,12}$. During this time all collected and stored data from the boulder collection operations is downlinked to Earth and the boulder mass properties are discerned through a series of small maneuvers. With the new mass properties of the system understood and all data downlinked, the ARV heads to a stand-off position in order to refine the asteroid orbit prior to the start of the EGT demonstration.

\section{Enhanced Gravity Tractor Operations and Control}

The gravity tractor concept was first proposed by Lu and Love in 2005 as a way to deflect the orbit of an asteroid using mutual gravitational force by hovering a large spacecraft in the vicinity of the asteroid ${ }^{13}$. A gravity tractor demonstration will be performed by placing the ARV (with the boulder) into a displaced, non-Keplerian, orbit (otherwise known as a halo orbit) about the velocity vector of the asteroid. Figure 8 shows the trajectory and control strategy where relative measurements will be taken by the MFOV camera. A preliminary covariance analysis was

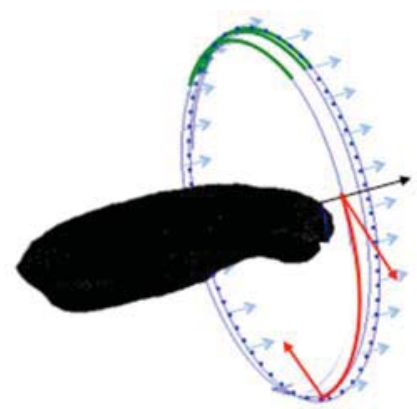

Figure 8. Enhanced Gravity Tractor operations and control strategy completed to estimate the navigation error, incorporating camera measurements as well as range, range rate, and Delta-DOR. Preliminary results are shown in Figure 9. The error converges to about $25 \mathrm{~m}$ and $0.3 \mathrm{~m} / \mathrm{s}$. Further work is being performed to increase the fidelity of the simulation, characterize the measurement error, and incorporate controller dispersions.

Continuous thrusting from the SEP system will be used to counteract the gravitational force imparted on the ARV by the asteroid and chemical impulses will be used to maintain the halo orbit. The ARV images will be taken once per orbit, while the ARV is at the top of the halo orbit. 

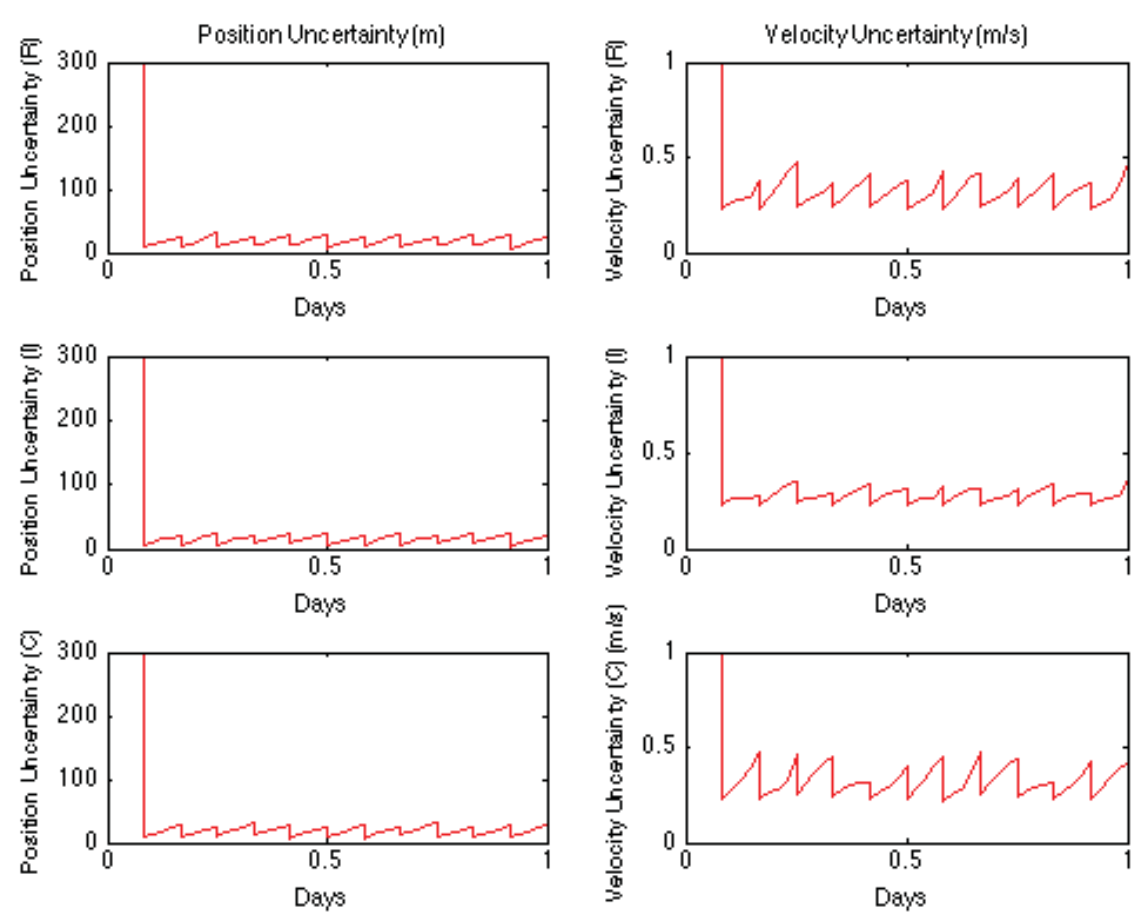

Figure 9. ARV relative position and velocity error

To model the SEP, a simple $\mathrm{x}$-axis PD controller was implemented, as proposed in by Wie,

$$
T_{i}=-K_{p}\left(x_{i}-d_{i}\right)-K_{d} \dot{x}_{i}
$$

where $K_{p}$ and $K_{d}$ are the gains, $d_{i}$ is the desired x-axis location, and $x_{i}$ and $\dot{x}_{i}$ are the x-axis position and velocity of the ARV with respect to the asteroid ${ }^{14}$. The trajectory was propagated with respect to an inertial frame attached to the asteroid (shown in Figure 10), neglecting the slow orbital motion of the NEA.

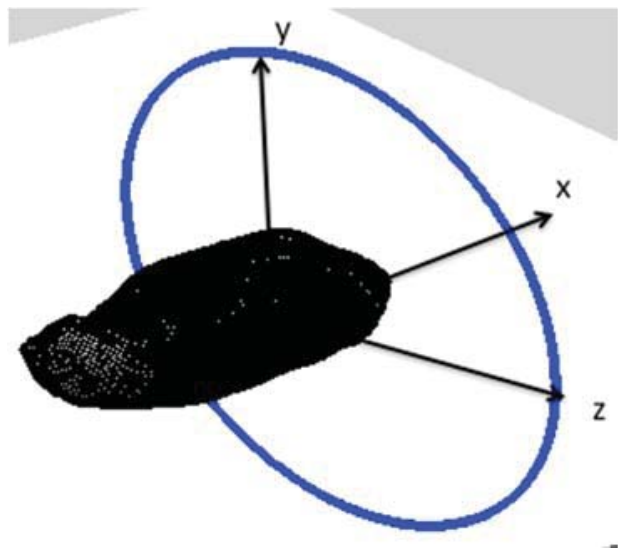

Figure 10. NEA centered frame. The coordinate frame used in the Enhanced Gravity Tractor analysis is defined as an asteroid centered inertial reference-frame, ignoring orbital motion. 
Table 3. Enhanced Gravity Tractor simulation parameters

\begin{tabular}{|c|c|c|}
\hline \multicolumn{3}{|c|}{ Simulation Parameters } \\
\hline Start Epoch & September 1,2020 00:00:00 & $\mathrm{kg}$ \\
\hline NEA Mass & $3.54 \times 10^{10}$ & $\mathrm{~kg}$ \\
\hline ARV Mass & 9020 & $\mathrm{~kg}$ \\
\hline Boulder Mass & 7000 & degrees \\
\hline Thruster Plume Angle & 45 & $\mathrm{hrs}$ \\
\hline Halo Orbit Period & 12 & \\
\hline
\end{tabular}

A numerical simulation was performed using Matlab's ode45 integrator. Control was applied only to the x-axis and limited to a range of 0.014 to $1.6 \mathrm{~N}$. shows the reference trajectory, in red, plotted on top of the actual trajectory. Note that
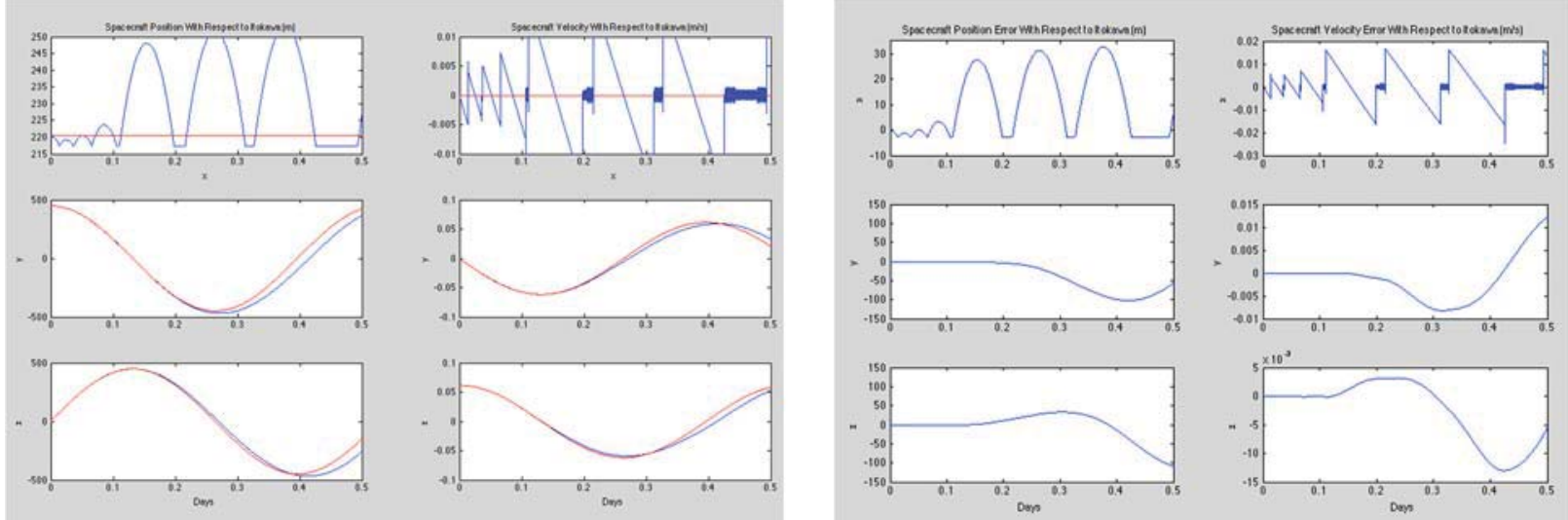

Figure 11. Controlled and reference positions and velocities
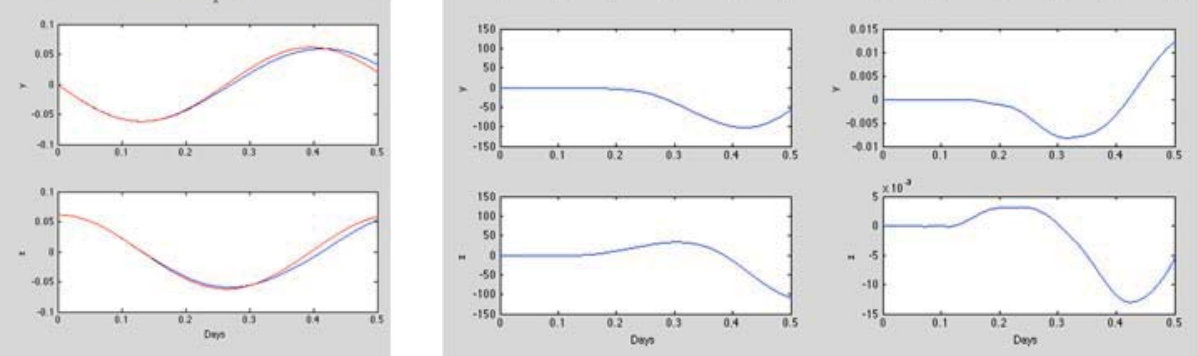

Figure 13. Position and velocity deviation from reference trajectory

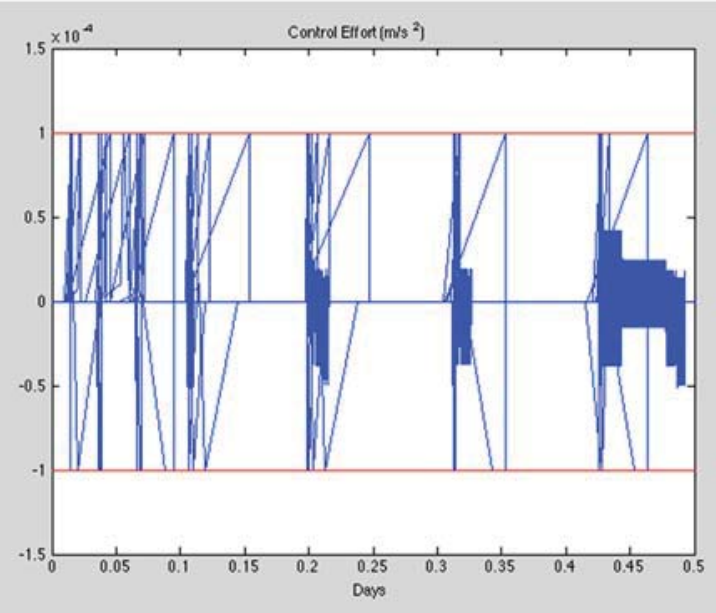

Figure 123. Required control effort 


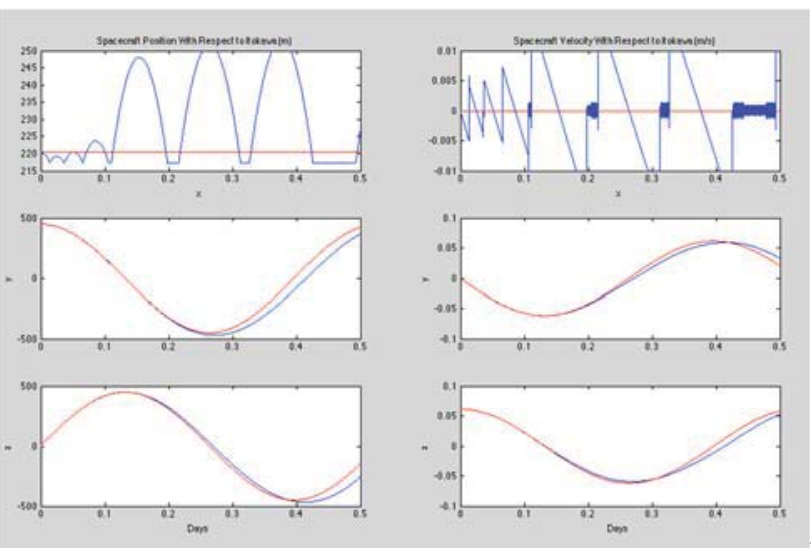

Figure 111 the $\mathrm{x}$-axes are scaled differently than $\mathrm{y}$ and $\mathrm{z}$.

Error! Reference source not found. 2 shows the deviation from the reference halo orbit and Error! Reference source not found. 3 shows the required control effort. The spacecraft is allowed to drift about a meter away from the nominal trajectory in the direction of the asteroid. The ARV is allowed to drift away and the gravity of the asteroid pulls the spacecraft back toward the nominal trajectory. Further linear covariance analyses and high fidelity Monte Carlo simulations are currently being performed to quantify the closed-loop system performance.

\section{Performance Results}

With the operations defined and the trajectories developed, a complete end-to-end proximity operations $\Delta \mathrm{V}$ budget was tracked and a propellant allocation was derived assuming the ARV weighs $9,000 \mathrm{~kg}$ at the start of the phase and the boulder that is collected weighs $8,000 \mathrm{~kg}$. The conservative $\Delta \mathrm{V}$ budget included a contingency allRCS ascent for all five boulder collection attempts, 180 days in the EGT orbit, and a $25 \%$ margin added to all values to allow for growth as both the mission and analysis mature. The RCS $I_{\mathrm{sp}}$ was assumed to be $230 \mathrm{~s}$ while the baseline SEP $\mathrm{I}_{\mathrm{sp}}$ of $3,000 \mathrm{~s}$ was scaled to account for inefficiencies due to the throttling profile required for the EGT demonstration.

Table 4 shows a summary of the developed budget and mission timeline broken down by event to show the detailed $\Delta \mathrm{V}$, duration, initial mass $\left(\mathrm{M}_{\mathrm{i}}\right)$, final mass $\left(\mathrm{M}_{\mathrm{f}}\right)$ and propellant used. The mission allocations for these operations were 400 days, $400 \mathrm{~kg}$ of RCS propellant, and $200 \mathrm{~kg}$ of xenon. While the duration and xenon allocations don't appear to have much room for growth, it must be noted that the developed budget is highly conservative and already accounts for contingency operations. If the first boulder collection attempt is success, an additional 50 days can be added back into the margin time. Similarly, the $\Delta \mathrm{V}$ for the EGT demo includes 180 days in the tractor orbit while it has been shown that only 60 days are required. This covers any variations in the orbit and any contingencies that would force the ARV out of this orbit for extended periods of time. 
Table 4. Initial $\Delta \mathrm{V}$ budget and propellant usage for Itokawa mission. Propellant numbers are calculated assuming a 9,000 kg ARV at the beginning of approach, an 8,000 kg boulder, RSC $I_{s p}$ of $230 \mathrm{~s}$, baseline SEP $I_{s p}$ of 3,000 s adjusted for lower throttling, and 25\% $\Delta \mathrm{V}$ margin.

\begin{tabular}{|l|c|c|c|c|c|c|c|}
\cline { 2 - 8 } \multicolumn{1}{c|}{} & $\begin{array}{c}\text { RCS DV } \\
(\mathbf{m} / \mathbf{s})\end{array}$ & $\begin{array}{c}\text { SEP DV } \\
(\mathbf{m} / \mathbf{s})\end{array}$ & $\begin{array}{c}\text { Duration } \\
(\text { days })\end{array}$ & $\begin{array}{c}\text { Mi } \\
(\mathbf{k g})\end{array}$ & $\begin{array}{c}\text { Mf } \\
(\mathbf{k g})\end{array}$ & $\begin{array}{c}\text { RCS Prop } \\
(\mathbf{k g})\end{array}$ & $\begin{array}{c}\text { SEP Prop } \\
(\mathbf{k g})\end{array}$ \\
\hline Approach & 12.500 & 0.000 & 19.0 & 9,000 & 8,950 & 49.7 & 0.0 \\
\hline Characterization & 16.479 & 0.000 & 32.0 & 8,950 & 8,885 & 65.2 & 0.0 \\
\hline Dry Run 1- Site 1 & 1.666 & 0.000 & 5.3 & 8,885 & 8,879 & 6.6 & 0.0 \\
\hline Dry Run 2 - Site 1 & 2.083 & 0.000 & 5.3 & 8,879 & 8,870 & 8.2 & 0.0 \\
\hline Boulder Collection 1 & 4.081 & 0.000 & 7.4 & 8,870 & 8,854 & 16.0 & 0.0 \\
\hline Boulder Collection 2 & 3.974 & 0.000 & 7.4 & 8,854 & 8,839 & 15.6 & 0.0 \\
\hline Dry Run 1- Site 2 & 1.647 & 0.000 & 5.3 & 8,839 & 8,832 & 6.5 & 0.0 \\
\hline Dry Run 2- Site 2 & 2.084 & 0.000 & 5.3 & 8,832 & 8,824 & 8.2 & 0.0 \\
\hline Boulder Collection 3 & 3.974 & 0.000 & 7.4 & 8,824 & 8,809 & 15.5 & 0.0 \\
\hline Boulder Collection 4 & 3.974 & 0.000 & 7.4 & 8,809 & 8,793 & 15.5 & 0.0 \\
\hline Dry Run 1- Site 3 & 1.647 & 0.000 & 5.3 & 8,793 & 8,787 & 6.4 & 0.0 \\
\hline Dry Run 2- Site 3 & 2.084 & 0.000 & 5.3 & 8,787 & 8,779 & 8.1 & 0.0 \\
\hline Boulder Collection 5 & 3.974 & 0.000 & 7.4 & 8,779 & 16,759 & 19.3 & 0.0 \\
\hline Planetary Defense & 5.343 & 114.550 & 261.7 & 16,759 & 16,523 & 39.3 & 196.7 \\
\hline Total & $\mathbf{6 5 . 5 1 0}$ & $\mathbf{1 1 4 . 5 5 0}$ & $\mathbf{3 8 1 . 1}$ & $\mathbf{9 , 0 0 0}$ & $\mathbf{1 6 , 5 2 3}$ & $\mathbf{2 8 0 . 1}$ & 196.7 \\
\hline
\end{tabular}

\section{Conclusions}

Through the extensive work performed by the ARRM Option B Proximity Operations Team, it has been shown that the concept of retrieving a boulder from the surface of an asteroid and demonstrating the Enhanced Gravity Tractor planetary defense technique is feasible and within the proposed capabilities of the ARV. While there are multiple uncertainties at this point in both the asteroid characteristics and the vehicle, the conservative and detailed approach in the above analysis allows for confidence in the design. With the launch of the ARRM currently set for 2017, on-going and future work will continue to investigate these uncertainties, refine the analysis, and update the operations as needed ${ }^{15}$.

\section{Acknowledgments}

The authors would like to thank the tremendous amount of work and support from the entire ARRM Option B Proximity Operations Team which this paper is built upon. Specifically we'd like thank Jay McMahon from the Colorado Center for Astrodynamics Research for his work on the Characterization phase trajectory development; Matt Strube from NASA Goddard Space Flight Center for his support with the sensor and optic capabilities; Shyam Bhaskaran from the Jet Propulsion Laboratory for his help in understanding the EGT deflection verification process and timing; Kenneth Getzandanner from NASA Goddard Space Flight Center for his help with the optical navigation model; and Haijun Shen and Dave Cornelius from Analytical Mechanics Associates and Carlos Roithmayr from NASA Langley Research Center for their support in the development of the terminal descent and ascent trajectories and controller.

\section{References}

${ }^{1}$ Mazanek, D., Brophy, and Merrill, R. G., “Asteroid Retrieval Mission Concept - Trailblazing Our Future in Space and Helping to Protect Us from Earth Impactors," $3^{\text {rd }}$ IAA Planetary Defense Conference, April 2013.

${ }^{2}$ Muirhead, B. "Asteroid Redirect Robotic Mission (ARRM) Refernce Mission Concept Study Public Information Package V1." August 20, 2013. URL: http://www.nasa.gov/sites/default/files/files/Asteroid-Redirect-Robotic-Mission-MuirheadTAGGED2.pdf. [cited 20 June 2014].

${ }^{3}$ Shinsuke, A., Tadashi, M., Naru, H., et al. "Mass and Local Topography Measurements of Itokawa by Hayabusa," Science, Vol. 312 No. 5778, 2 June 2006, pp. 1344-1347. 
${ }^{4}$ Mazrouei, S., Daly, M. G., Barnouin, O. S., Ernst, C. M., and DeSouza, I., "Block Distributions on Itokawa,” 2014. Icarus, Issue 229, p 181-189.

${ }^{5}$ Merrill, R. G., Qu, M., Vavrina, M. A., and Englander, J. A. "Interplanetary Trajectory Design for the Asteroid Robotic Redirect Mission Alternate Concept Trade Study.” AIAA Space Conference, Aug. 2014.

${ }^{6}$ Merrill, R. G. and Belbin, S. P. "Boulder Capture System Design Operations for the Asteroid Robotic Redirect Mission Alternate Concept Study.” AIAA Space 2014 Conference, Aug. 2014.

${ }^{7}$ Scheeres, D.J., Gaskell, R., et. al. "The Actual Dynamical Environment About Itokawa." AIAA/AAS Astrodynamics Specialist Conference and Exhibit, AIAA 2006-6661, August 2006.

${ }^{8}$ R. W. Gaskell. "Optical Navigation Near Small Bodies.” AAS/AIAA Space Flight Mechanics Meeting. AAS 11-220, 2011.

${ }^{9}$ Galante, J., Van Eepoel, J., Strube, M., Gill, N., Gonzalez, M., Hyslop, A., and Partrick, B. "Pose Measurement Performance of the Argon Relative Navigation Sensor Suite in Simulated Flight Conditions." AIAA Guidance, Navigation and Control Confernce, CP6845, Aug. 2012.

${ }^{10}$ Owen, W. M. Jr. "Methods of Optical Navigation,” AAS Spaceflight Mechanics Conference, AAS 11-215, Feb. 2011.

${ }^{11}$ Shen, H., Roithmayr, C. M., and Cornelius, D. M. "Controlled Ascent from the Surface of an Asteroid", AIAA Astrodynamics Specialist Conference, Aug. 2014.

${ }^{12}$ Shen, H., Roithmayr, C. M. "Co-spin with Symmetry Axis Stabilization, and De-spin for Asteroid Capture", American Control Conference, June 2014.

${ }^{13} \mathrm{Lu}$, E. T., and Love, S. G. “Gravitational Tractor for Towing Asteroids.” Nature, Vol. 438. Nov. 10, 2005, pp. 177, 178.

${ }^{14}$ Wie, B, "Dynamics and Control of Gravity Tractor Spacecraft for Asteroid Deflection." Journal of Guidance, Control, and Dynamics, Vol. 31. No.5. Sept.-Oct. 2008, pp. 1413-1423.

${ }^{15}$ Lightfoot, R. "NASA's FY2014 Asteroid Strategy: An Integrated Strategy in Support of Human Exploration and Protection of the Planet." April 10, 2013. URL: http://www.nasa.gov/pdf/740684main_LightfootBudgetPresent0410.pdf. [cited 20 June 2014]. 